\section{1}

2

3

4

5

6

1

2

\title{
A Toolkit for Analyzing Strategy Use in Phonemic Verbal Fluency Tasks
}

\author{
Brandon C. Mackey ${ }^{1}$, Sara A. Sims ${ }^{1}$, David E. Vance ${ }^{2}$, and Kristina M. Visscher ${ }^{3}$
}

${ }^{1}$ Department of Psychology, University of Alabama at Birmingham

${ }^{2}$ School of Nursing, University of Alabama at Birmingham

${ }^{3}$ Department of Neurobiology, University of Alabama at Birmingham

Author Note

Brandon C. Mackey: https://orcid.org/0000-0001-6378-6029

David E. Vance: https://orcid.org/0000-0002-0498-6263

Sara A. Sims: https://orcid.org/0000-0001-7107-1891

Kristina M. Visscher: https://orcid.org/0000-0003-0737-4024

The data used for the analysis described in this article were obtained from a dataset of

260 individuals, which was originally collected by Vance et al. as part of a longitudinal clinical

trial that examined the effect of a computerized cognitive training protocol on cognitive

21 functioning in middle-aged and older adults (40+) who had been diagnosed with HIV (Vance et

22 al., 2017). The collection of that data was supported by funding from the Edward R. Roybal

3 Center (P30 AG022838), a National Institutes of Health NIA K-award (PI: Fazeli: 
24 K99AG048762) titled "A Novel Neurorehabilitation Approach for Cognitive Aging with HIV,"

25 and a National Institutes of Health NIMH R01-award (1R01MH106366-01A1) titled "An RCT

26 of Speed of Processing Training in Middle-aged and Older Adults with HIV." The authors of this

27 article have read and approved this final version of the manuscript and have no known conflicts

28 of interest to disclose.

29 Correspondence concerning this article should be addressed to Brandon C. Mackey;

30 email: bmackey@uab.edu. 
Abstract

The phonemic verbal fluency task is a common cognitive assessment of language and

33 executive functioning which asks participants to list as many words as they can that begin with a

34 given letter. Verbal fluency tasks are widely used to identify deficits in verbal fluency, which

35 have been associated with disorders such as schizophrenia and dementia. Verbal fluency tasks

36 are scored by the number of correct responses, however analysis of "clusters" of related words

37 within a response list can give insights into the cognitive strategies used by participants.

38 Unfortunately, manual word cluster analysis is time and labor intensive and inconsistent, since

39 raters may cluster words differently depending on how they themselves have phonetically

40 categorized the words. We present an automated pipeline for quantification of strategy use in the

41 phonemic verbal fluency task, "LetterVF". LetterVF is a python module (i.e., a script containing

42 useful functions, which can be imported and used in other scripts) that uses a pronunciation

43 dictionary to convert verbal fluency task data items into lists of phonemes, which can be

44 analyzed to identify clusters of words that share similarities in any of several clustering

45 categories. Additionally, LetterVF contains useful functions for identifying intrusions (words

46 which do not follow the rules for the task), identifying perseverations (responses repeated within

47 the same trial), counting the number of cluster switches in a list, and calculating the average size

48 of clusters for a list. Analysis of data from 50 participants' verbal fluency task responses

49 indicated that analysis using LetterVF yields accuracy and consistency on par with manual

50 analysis. Our hope is that this tool will allow researchers to get more out of their datasets, and

51 explore new topics related to cognitive strategy use, such as how strategies change with age and

52 differences in strategies between experimental groups. 
54 Key words: verbal fluency, phonemic verbal fluency task, python, strategy

55 Tools: Python 


\section{Introduction}

The verbal fluency task (VFT) has been one of the most common methods among researchers and clinicians for measuring language, verbal fluency, and executive functioning. A major reason for its wide-spread use is that it is an easy-to-administer test, which in a matter of minutes can give data related to a patient's ability to retrieve information from their memory

61 (Bousfield \& Sedgwick, 1944). During a fluency task, a participant is given a category and asked to list words from that category within a given time limit (usually one minute). There are two

63 types of verbal fluency tasks: semantic verbal fluency tasks, in which participants are given a

64 semantic category (e.g., animals), and phonemic verbal fluency, in which participants are given a

65 letter with which all their responses must begin. Verbal fluency tasks are commonly used in the

66 study of clinical disorders such as schizophrenia (Ojeda et al., 2010), Alzheimer's disease

67 (Pakhomov \& Hemmy, 2014), and Parkinson's disease (Koertz et al., 2012). Additionally, verbal

68 fluency tasks are included in popular cognitive screening batteries such as the Montreal

69 Cognitive Assessment (MoCA) (Nasreddine et al., 2005).

71 data is richly structured, in that additional information can be collected from the same data set

72 when researchers consider the order in which participants produce their responses and how they

73 do so. By analyzing how consecutive responses relate to one another, it is possible for

74 researchers to gain insight into the cognitive strategies that participants use during the task

75 (Troyer, Moscovitch, \& Winocur, 1997). For example, if a participant consecutively gives the

76 responses "fit", "fight", and "foot", they have produced a cluster of related words which

77 indicates that this participant may be using a strategy in which they change only a single vowel 
78 sound. Understanding what cognitive strategies participants use and how they use them makes it

79 possible for researchers to identify differences between individuals' performances.

80 Doing this type of word cluster analysis by hand, however, is time consuming and

81 inconsistent. Word cluster analysis is time consuming because it requires a trained scorer to

82 determine, item by item, the relationships between adjacent words in several categories. And

83 word cluster analysis is inconsistent because many of those relationships are defined

84 subjectively. For example, "Cot" and "caught" are pronounced in exactly the same way in some

85 parts of the United States, but have distinct pronunciations in other places. Researchers may

86 differ over whether or not they believe these words rhyme, and in any case it is sometimes

87 impossible to consider all of the possible pronunciations and spellings of words. Some methods

88 have been developed for automating word cluster analysis, however these efforts have primarily

89 focused on automating the cluster scoring of semantic verbal fluency tasks (Shao et al., 2014;

90 Zemla et al., 2020). Because there is not a common and fast way to complete cluster analyses on

91 phonemic verbal fluency tasks, researchers are missing valuable information about the phonemic

92 VFT. Here we present LetterVF.py, a python module (i.e., a python script which contains

93 functions that can be imported into and used in other scripts) which contains functions for

94 automating word cluster analysis of phonemic VFT data.

\section{Method}

\section{Software Accessibility}

97 LetterVF is free to use and modify according to the MIT license (Open Source Initiative,

98 n.d.). LetterVF can be downloaded from https://github.com/Visscher-Lab/LetterVF, or by using

99 the command python3 -m pip install "LetterVF". LetterVF has functions that draw from

100 the numpy.py and xlsxwriter.py python modules. If the user does not have either of these 
101 packages installed and updated, installing LetterVF using pip will automatically install or update

102 them. LetterVF was developed to be compatible with the formatting of CMUdict, which is an

103 open-source North American English pronunciation dictionary created by researchers at

104 Carnegie Mellon University (The Carnegie Mellon Speech Group, 1998). As such, if you will be

105 using LetterVF for analyzing lists in North American English, we recommend using CMUdict

106 with LetterVF, though any pronunciation dictionary will work so long as it is formatted as a

107 plain text file where each line contains a single word followed by its phonemes, separated by

108 spaces. CMUdict can be downloaded from their project's website

109 (http://www.speech.cs.cmu.edu/cgi-bin/cmudict), or by using the terminal command python3

110 m pip install cmudict.

\section{Formatting Datasets}

112 LetterVF is designed to analyze a 3-column CSV file, where the first column contains a

113 participant identification number, the second column contains a list identification number (for

114 use cases where participants have multiple lists being analyzed at once), and the third column

115 contains the responses given by the participant. The first row should act as a label for each

116 column (e.g., the first cell of the first row might contain "Participant Identification Number" to

117 make it clear for the user what kind of information is represented in each column. An example of

118 what the data file should look like is in Figure 1. The participant identification numbers and list

119 numbers can be formatted however the user prefers (i.e., can be codes containing letters as well),

120 as long as the formatting is consistent. 


\begin{tabular}{|r|l|}
\hline participant_id & list_number response \\
\hline 1 & 1 freckle \\
\hline 1 & 1 fry \\
\hline 1 & 1 fly \\
\hline 1 & 2 dolt \\
\hline 1 & 2 fair \\
\hline 1 & 2 fare \\
\hline 1 & 2 fall \\
\hline 2 & 1 flack \\
\hline 2 & 1 full \\
\hline 2 & 1 fetter \\
\hline 2 & 1 ferret \\
\hline 2 & 1 fend \\
\hline 2 & 1 faberge \\
\hline
\end{tabular}

Figure 1. Example of how a dataset .csv file should be formatted for use with lettVF.py.

\section{Python Module}

124 A pronunciation dictionary is used to look up and compare phonemes that are used for

125 each word's pronunciation in order to analyze phonemic verbal fluency task data. Once a user has

126 downloaded the LetterVF.py according to the instructions in the Software Accessibility section,

127 and a properly formatted data file and pronunciation dictionary have been created, LetterVF.py

128 can be used to analyze the data according to any one of several clustering algorithms that are

129 included in LetterVF.py. Figure 2 shows a basic overview of how LetterVF processes phonemic

130 verbal fluency task data. 


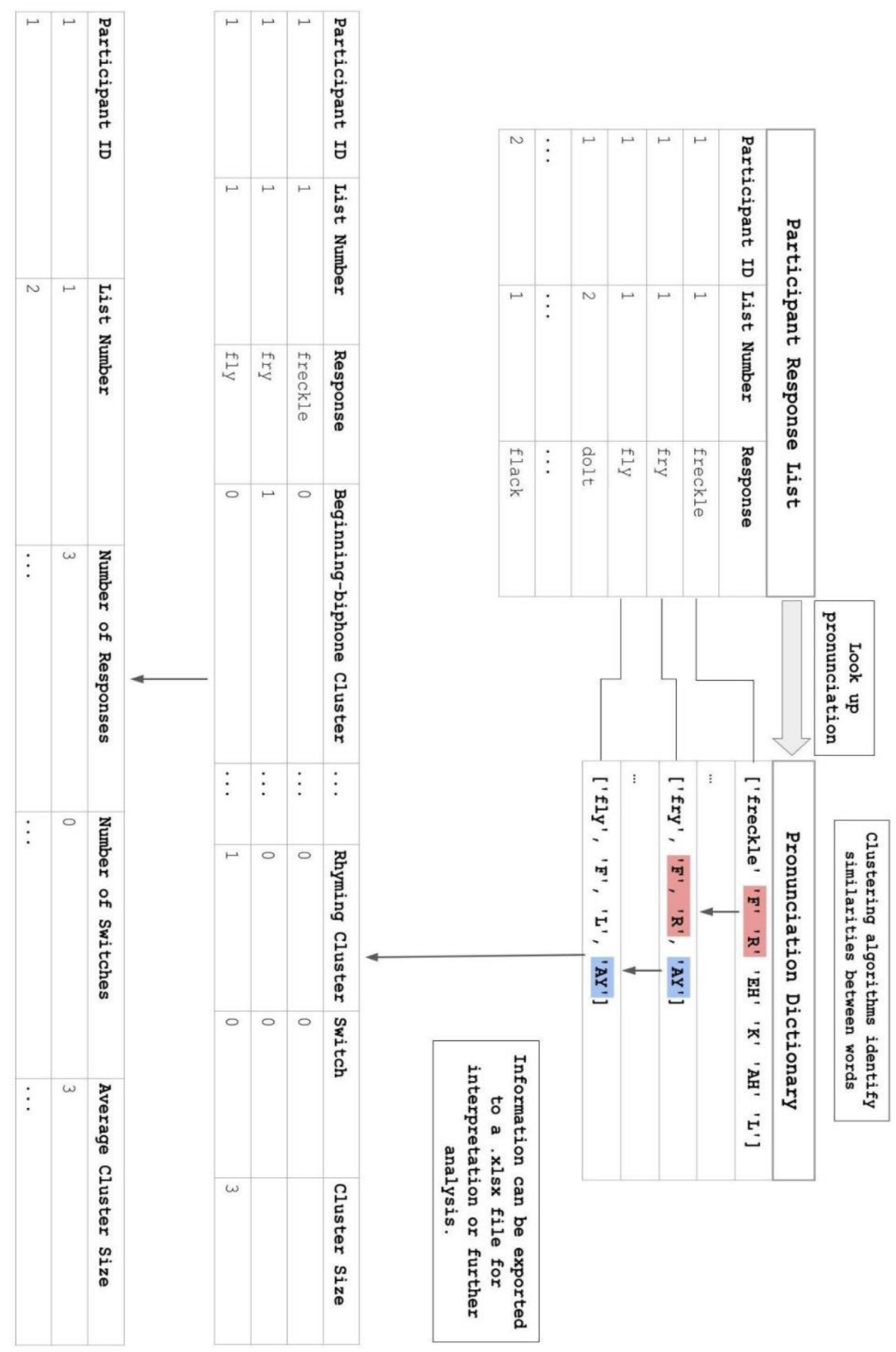
tasks. 


\section{Step \#1: Importing LetterVF and setting up pronunciation dictionary} import LetterVF as lvf should be included at the top of a user's Python script in order to import all functions from LetterVF. The first step is to use lvf.getPronDict(). This function sets up the user's pronunciation dictionary for analysis. The first argument of lvf.getPronDict() is character, and it should be a string set to the target letter for the lists being analyzed (e.g., "f" for lists of words beginning with "f"). The second argument, pron_dict_path, should be a string which contains the pronunciation dictionary's file path. If the verbal fluency task being analyzed had restrictions on the types of words allowed (e.g., not allowing proper nouns), the user may want to utilize the third, optional parameter of lvf.getPronDict(), which is removal_list_path. This third parameter should be set to a string which contains the path for a file which lists any words to be removed from the pronunciation dictionary. This file can be either a csv or text file which contains on each line one word to be removed. The fourth and final argument to lvf.getPronDict(), addendum_path, is also optional, but useful for adding words to the pronunciation dictionary. This is useful in cases where the pronunciation dictionary being used does not contain entries for all valid responses given by participants. Setting addendum_path to a string containing the path to an addendum file will add any entries in that file to the pronunciation dictionary. An addendum file should be a csv file formatted so that each line contains a single word, followed by each of the phonemes of that word in separate cells. An example of what this addendum file should look like can be found

\section{in Figure 3.}

In order to determine which phonemes to use for a given word, the user can sound out the word and assign phonemes based on their own judgement. CMUDict uses 2-letter ARPAbet symbols, a guide to which can be found on the project's website (The Carnegie Mellon Speech 
158 Group, 1998). (ARPAbet symbols typically also include numbers after some symbols to indicate

159 which syllables are stressed; however, these are unnecessary when creating files for use with

160 LetterVF.) The current version of LetterVF does not take into account the stresses on different

161 syllables, so it is not necessary to include any numbers for identifying stresses.

162 lvf.getPronDict() will return a list of strings, where each string is an item from the

163 pronunciation dictionary which begins with the target letter. For words that have multiple

164 pronunciations listed in the dictionary, the first pronunciation to appear in the dictionary is the

165 one that will be used during analysis. An example of how to use lvf.getPronDict() can be

166 found in Listing 1.

167

168

169

170

171

172

173

174

175

176

177

178

179

180

181

182

183

184

185

186

187

188

189

190

\begin{tabular}{|c|c|c|c|c|c|c|c|c|c|c|c|c|}
\hline$\triangle$ & A & B & C & D & $E$ & $\mathrm{~F}$ & G & $\mathrm{H}$ & 1 & $J$ & K & L \\
\hline 1 & fibber & $\mathrm{F}$ & $\mathrm{IH}$ & B & ER & & & & & & & \\
\hline 2 & floozy & $\mathrm{F}$ & L & UW & Z & IY & & & & & & \\
\hline 3 & frugivore & $\mathrm{F}$ & $\mathrm{R}$ & UW & G & $\mathrm{AH}$ & V & OW & ER & & & \\
\hline 4 & folivore & $\mathrm{F}$ & OW & $\mathrm{L}$ & $\mathrm{OH}$ & V & OW & ER & & & & \\
\hline 5 & finial & $\mathrm{F}$ & IH & $\mathrm{N}$ & IY & UH & L & & & & & \\
\hline 6 & forementioned & $\mathrm{F}$ & OW & ER & $M$ & IH & $\mathrm{N}$ & $\mathrm{CH}$ & $\mathrm{Y}$ & $\mathrm{AH}$ & $\mathrm{N}$ & D \\
\hline
\end{tabular}

Figure 3. Example of how .csv files should be formatted if the user wishes to add additional words to their preferred pronunciation dictionary.

Listing 1. Preparing a pronunciation dictionary using LetterVF

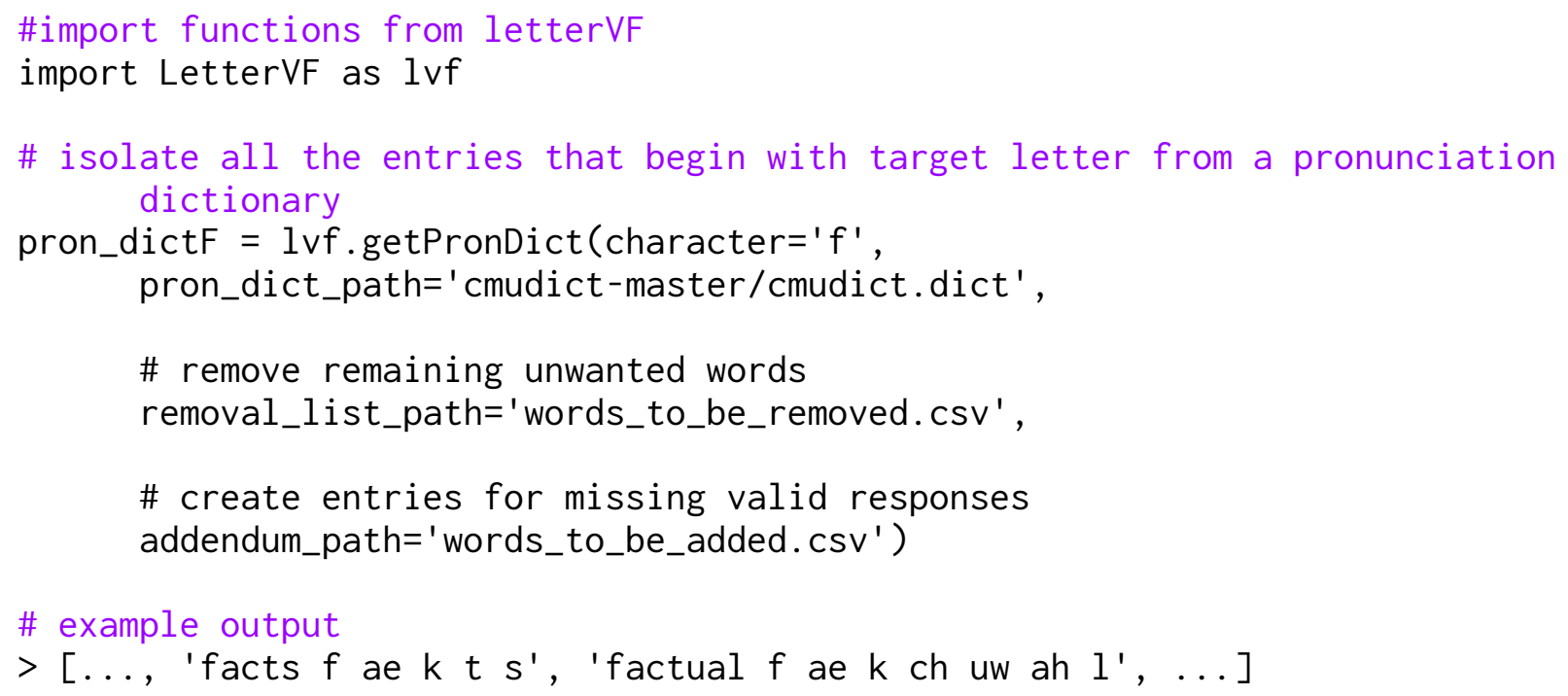


Step \#2: Identifying words missing from the pronunciation dictionary

The second step is optional but recommended. lvf. findIntrusions() takes two arguments: pron_dict should be set to a pronunciation dictionary in the form of a list of strings (e.g., a list returned by lvf.getPronDict()), and data_file_path should be set to a string containing a data file path. This function returns a list of all of the intrusions that LetterVF could identify in the data file. Intrusions are any items in the data file that do not have a corresponding entry in the pronunciation dictionary. As such, valid responses may be marked as intrusions any time the pronunciation dictionary being used does not contain an entry for that response. In order to check whether your pronunciation dictionary contains entries for all valid responses in the data file, we recommend using lvf.findIntrusions() on the initial pronunciation dictionary to identify any valid responses that are incorrectly being identified as intrusions, then creating an addendum file and including it in the second iteration of the pronunciation dictionary. Listing 2 shows how to find and show all intrusions found in a data file.

\section{Listing 2. Using lvf.findIntrusions() to identify intrusions in a dataset}

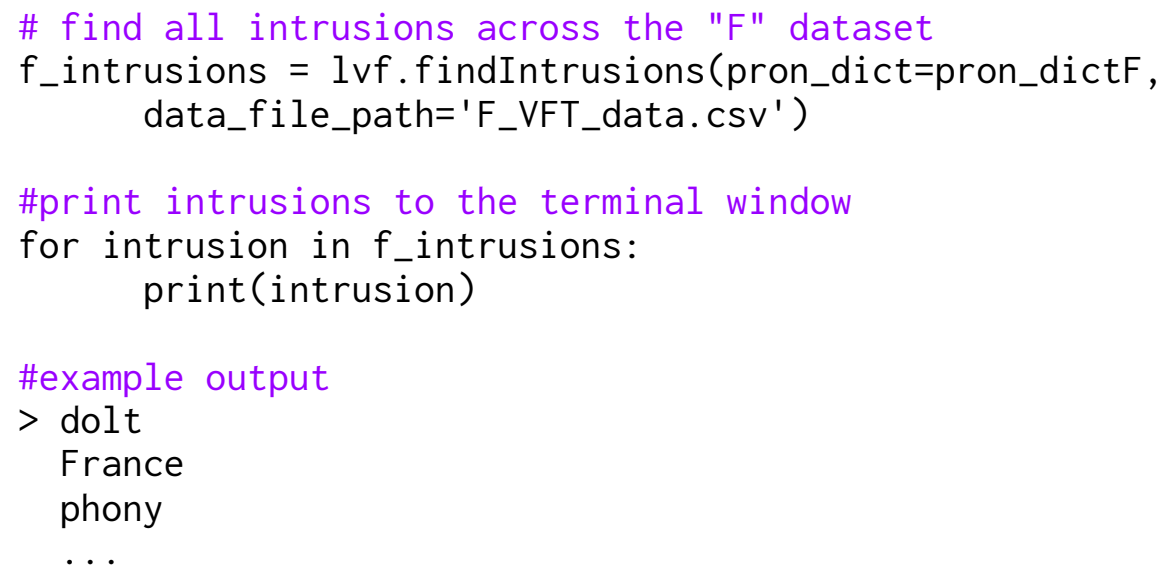



242 that it begins with "fl", because it rhymes with "fry". The first item of each list is not considered 243 a switch. Perseverations can cluster with adjacent words, but intrusions cannot. Clusters of size 1 244 are included, and intrusions are considered clusters of size 1.

\section{Step \#3: Identifying intrusions and perseverations and calculating clusters}

Once the user has a pronunciation dictionary that contains all and only the words which should be counted as valid, the third step involves using lvf.findClusters() to create an array which contains all of the data items and how they relate to the preceding item in each clustering category included. The first two parameters of lvf.findClusters() are pron_dict and data_file_path; these parameters behave exactly the same as they do in lvf.findIntrusions(). There are seven more optional parameters, and each one determines whether or not to include a specific clustering category in the clustering analysis. Table 1 shows these parameters and explains what clustering category they represent. The clustering categories that are set to be included by default are include_rhyme=True, first_letters $=2$, include_homophone=True, and lev_dist $=1$. These categories were selected to be included by default because they are commonly used for scoring clusters (Troyer et al., 1997). In addition to scoring consecutive words according to each of the selected clustering algorithms, this function will automatically mark intrusions (i.e., invalid responses), mark perseverations (i.e., words that are repeated within a list), mark switches (i.e., where new clusters begin), and calculate the size of each cluster. Switches are considered to occur only when a word does not form a cluster with the previous word in any of the included clustering categories. For example, in Listing $\mathbf{3}$, lvf.findClusters() counts "freckle" and "fry" as part of the same because they share the same two first letters. However, "fly" is also considered a part of the same cluster despite the fact 
246 Listing 3. Using lvf.findClusters() to identify words that group together according to the 247 clustering algorithms included in analysis

\# find clusters, switches, intrusions, perseverations, and cluster sizes for each list

clust_listF $=$ lvf.findClusters(prond_dict=pron_dictF, data_file_path='F_VFT_data.csv')

\#example output

$>\left[\left[{ }^{\prime} 1\right.\right.$ ', ' 1 ', 'freckle' $, 0,1,0,0,0,1$ ' $]$,

$\left[' 1{ }^{\prime}, ' 1\right.$ ', 'fry' $, 0,1,0,0,0, '$ '],

['1', '1', 'fly', $1,0,0,0,3]$,

['1', '2', 'dolt' , 'intrusion' , 'intrusion ', 'intrusion' , 'intrusion' , 1, 1],

['1', '2', 'fair', $0,0,0,0,1,{ }^{\prime}$ ']

$[$ '1', '2' , 'fare' $, 1,1,1,1,0,2]$

...] 


\section{Table 1}

List of all optional parameters for the function IVf. findClusters(), as well as the default value of each parameter and a description of the clustering category that corresponds with each. The clustering categories included by default are include_rhyme, first_letters, include_homophone, and lev_dist.

\begin{tabular}{|c|c|c|}
\hline Parameter & Default Value & $\begin{array}{l}\text { Will cluster any } 2 \\
\text { consecutive words which... }\end{array}$ \\
\hline include_rhyme & True & $\begin{array}{l}\text { share a common final syllable } \\
\text { (i.e., syllabic rhyme) }\end{array}$ \\
\hline first_letters & 2 & $\begin{array}{l}\text { share the same first } n \text { letters, } \\
\text { where } n \text { is the number } \\
\text { assigned to the parameter }\end{array}$ \\
\hline include_homophone & True & $\begin{array}{l}\text { share the exact same } \\
\text { pronunciation }\end{array}$ \\
\hline lev_dist & 1 & $\begin{array}{l}\text { can be transformed into one } \\
\text { another by deleting, replacing, } \\
\text { or inserting } n \text { phonemes, } \\
\text { where } n \text { is the number } \\
\text { assigned to the parameter }\end{array}$ \\
\hline include_beg_biphone & $\begin{array}{l}\text { False (not included in } \\
\text { analysis by default) }\end{array}$ & $\begin{array}{l}\text { share the same first } 2 \\
\text { phonemes }\end{array}$ \\
\hline include_end_biphone & $\begin{array}{l}\text { False (not included in } \\
\text { analysis by default) }\end{array}$ & $\begin{array}{l}\text { share the same last } 2 \\
\text { phonemes }\end{array}$ \\
\hline edit_dist & $\begin{array}{l}\text { None (not included in } \\
\text { analysis by default) }\end{array}$ & $\begin{array}{l}\text { can be transformed into one } \\
\text { another by deleting or } \\
\text { replacing, } n \text { phonemes, where } \\
n \text { is the number assigned to the } \\
\text { parameter }\end{array}$ \\
\hline
\end{tabular}


271 intrusions or pereverations. Average cluster size is calculated by dividing the total number of

272 responses, including intrusions and perseverations, by the number of clusters. An example of

273 how to use lvf.clustSummary() can be seen in Listing 4.

274 Step \#5: Exporting to Excel file

276 separate sheets for the word cluster analysis and summary, the user can use lvf. sumExport().

277 This function has the parameters clust_list, which should be set to the array that resulted from

278 lvf.findClusters(), clust_sum, which should be set to the array resulting from

279 Ivf.clustSummary (), and output_path which should be set to a string that names where the

280 xlsx file should be saved and what it should be saved as. Be sure to end the file name with the

281 .xlsx extension. Listing 4 also shows how to use lvf. sumExport. 
Listing 4. Using lvf.clustSummary() to calculate the number of valid responses, number of switches, and average cluster size for each list in a dataset, then using lvf. sumExport () to export analysis to a .xlsx file for ease of interpretation.

\# find the number of valid items, number of switches, and average cluster sizes for each list

\section{Participants and Procedures}

In order to validate this new method for performing cluster analysis on phonemic verbal fluency task data, response lists to the Controlled Oral Word Association Test (COWAT) were analyzed. These response lists come from a dataset of 260 individuals, which was originally collected by Vance et al. as part of a clinical trial that examined the effect of a computerized cognitive training protocol on cognitive functioning in people who were at least 40 years old and who had been diagnosed with HIV (Vance et al., 2017). The participants included in the 260participant data set were recruited by placing flyers and other recruitment materials in a university HIV/AIDS clinic in an urban area in the Southern United States. The average age of the participants was 51.15 years old ( $S D=6.76$, including ages ranging from 40 to 73 ). In this sample, $17.31 \%$ of the participants identified themselves as white and $64.23 \%$ identified as male ( $82.69 \%$ identified as non-white, $35.77 \%$ identified as female). The average number of years of education of the participants was 12.53 years $(S D=2.24)$. 
Cluster analysis was performed on COWAT response lists from 50 participants at

313 baseline. Each participant completed three verbal fluency tasks, one for the letter "F", one for

314 "A", and one for "S".

315 First, the three lists from the 50 participants were scored manually. Items were

316 considered a part of a cluster if the word and the preceding word either shared a common final

317 syllable (i.e., syllabic rhyme), began with the same first two letters, the pronunciation of one

318 word was different from the pronunciation of the other by only 1 phoneme, or the words were

319 homophones. Names, proper nouns, and proper adjectives were considered intrusions, even if

320 they began with the target letter. No intrusions were counted in clusters with other words. Words

321 that did not cluster with other words, including intrusions, were considered to be clusters of size

322 1. Switches were defined between any two clusters, and the first response of each list was not

323 counted as a switch.

324 The number of valid responses, number of switches, and average cluster size were

325 calculated for each list. Perseverations were not counted as valid responses, but they were

326 counted in clusters with adjacent words if they matched in any of the clustering categories.

327 Average cluster size was calculated by dividing the number of switches by the number of

328 responses (including intrusions and perseverations).

329 An equivalent to the hand-scored set of values was then completed using functions from

330 LetterVF.py, according to the instructions and advice provided in the Python Module section

331 above. For this analysis, the CMUDict pronunciation dictionary was used to get a list of

332 phonemes for each word. 
Percentage agreements and correlations were then calculated for the manual- and

334 machine-found values for number of valid items, number of switches, and average cluster size

335 across all "F", "A", and "S" response lists.

\section{Results}

Percentage agreements ranged from $76 \%$ to $100 \%$. Correlation coefficients ranged from

3380.955 to 1.00 . Percentage agreements and correlation coefficients were highest across all letters

339 for number of valid items, were slightly lower for the number of switches, and slightly lower

340 again for the average cluster sizes. Percentage agreements and correlation coefficients were

341 similar across "F," "A," and "S"; however, these values were slightly lower for "A." All $p$-values

342 for correlations were less than 0.001. Table 2 shows the percentage agreements and correlation

343 coefficients between the hand-calculated scores and machine-calculated scores associated with

344 the number of valid items produced by participants, the number of switches between word

345 clusters in each response list, and the average cluster size for each response list for each letter.

346 Figure 4 shows the plot of the hand-calculated scores versus the machine calculated scores for

347 the number of valid items produced by participants, the number of switches between word

348 clusters in each response list, and the average cluster size for each response list, again for each

349 letter. 


\section{$351 \quad$ Table 2}

Calculated percent agreements and Pearson's correlation coefficients for the number of valid items, number of switches, and average cluster sizes in manually-scored versus machine-scored trials.

\begin{tabular}{|c|c|c|c|c|c|c|}
\hline & Number of & alid Items & Number of & witches & Average clu & ter size \\
\hline & $\begin{array}{l}\% \\
\text { Agreement }\end{array}$ & Pearson's r & $\begin{array}{l}\% \\
\text { Agreement }\end{array}$ & Pearson's r & $\begin{array}{l}\% \\
\text { Agreement }\end{array}$ & Pearson's r \\
\hline $\mathrm{F}$ & 100 & 1.00 & 82 & 0.993 & 82 & 0.978 \\
\hline A & 96 & 0.999 & 78 & 0.985 & 76 & 0.955 \\
\hline S & 98 & 0.999 & 82 & 0.988 & 80 & 0.969 \\
\hline
\end{tabular}




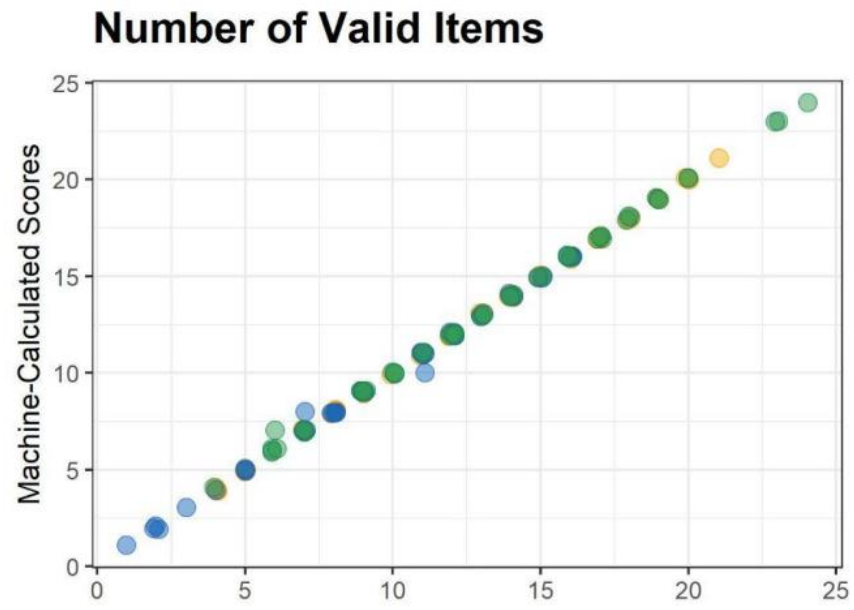

Number of Switches

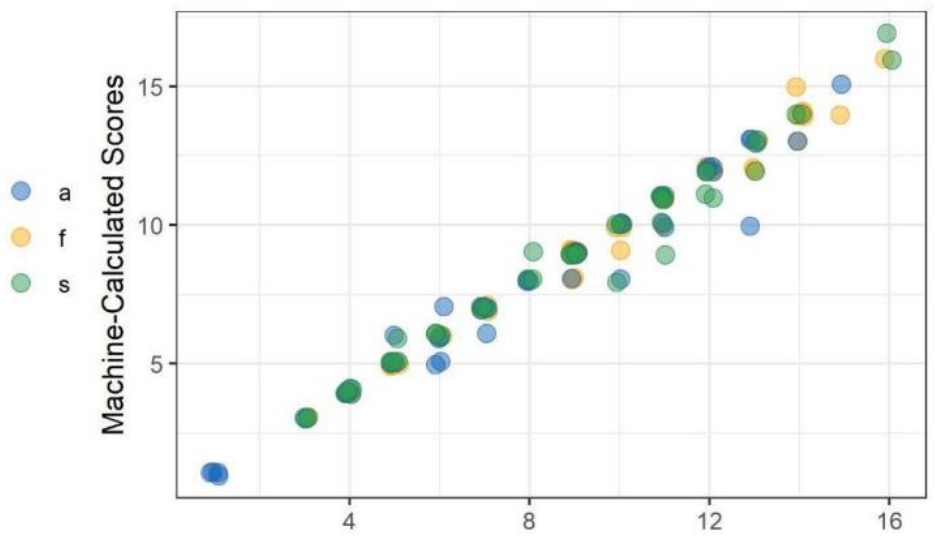

\section{Average Cluster Size}

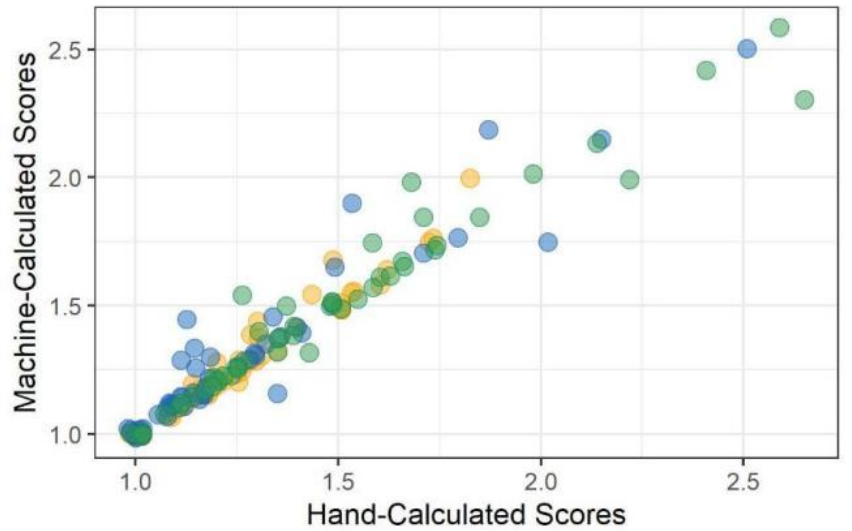

354 Figure 4. Scatterplots of hand-calculated scores versus machine-calculated scores for the number 355 of valid items identified, number switches, and average cluster size of each list. 


\section{Discussion}

The results suggest that using LetterVF.py to analyze phonemic verbal fluency task data

360 produces results similar to results produced by human scorers using equivalent scoring criteria.

361 There was a significant and high correlation between the values found by the human scorer and

362 the values found using LetterVF.py across all 9 measures. Lower percentage differences in

363 number of switches and average cluster size are likely due to the fact that these measures are

364 more subjective than the count of valid responses. Differences in results between the two

365 methods could be due to several factors, most likely related to human error and differences in the

366 pronunciation listed in the dictionary and the pronunciation used by the human scorer.

367 This test of LetterVF.py was limited by the fact that only one human scorer was included

368 in manual analysis, and by the fact that this experiment only tested the ability of LetterVF.py to

369 analyze "F", "A", and "S". LetterVF.py could be further validated in a similar study which uses

370 multiple human scorers and compares the inter-rater reliability among human scorers with an

371 inter-rater reliability between LetterVF.py and human scorers.

372 Our hope is that LetterVF.py may serve as an easy-to-use, flexible, and fast tool for

373 researchers to use for cluster analyses of phonemic fluency data. By making VFT cluster analysis

374 more accessible, we hope that researchers will have more opportunities to investigate

375 understudied topics, such as differences in cognitive strategies between experimental groups, or

376 how interventions may affect longitudinal changes in cognitive strategies within experimental

377 groups. The clinical implications of LetterVF.py will be unknown until such studies are done,

378 but it is our hope that analyses using LetterVF.py will be useful in cases where phonemic VFT

379 data is already collected and used (e.g., in assessing people with Alzheimer's disease or

380 Parkinson's disease). 
In the future, we hope to update and expand LetterVF.py so that it remains relevant and

382

383

384

385

useful for researchers. This may include improvements in the efficiency of the functions

contained within LetterVF.py, the addition of clustering categories for users to pick from, and

the addition of methods for identifying semantic relationships between data items (e.g. methods

that can cluster "finch" and "falcon" based on the fact that they are both birds).

\section{Acknowledgements}

We thank Jun Yeong Byun (University of Alabama at Birmingham) for her help in preparing the data used for this article. We also thank the members of the Visscher Lab for their feedback and suggestions throughout the development of this project.

\section{References}

Bousfield, W. A., \& Sedgewick, C. H. (1944). An analysis of sequences of restricted associative responses. The Journal of General Psychology, 30(2), 149-165.

https://doi.org/10.1080/00221309.1944.10544467

Carnegie Mellon Speech Group (1998). The CMU pronouncing dictionary. (n.d.). Retrieved September 1, 2021, from http://www.speech.cs.cmu.edu/cgi-bin/cmudict.

Gilleland, M. (2009). Levenshtein distance, in three flavors. Levenshtein Distance. Retrieved September 24, 2021, from http://people.cs.pitt.edu/ kirk/cs1501/Pruhs/Spring2006/assignments/editdistance/Levens htein\%20Distance.htm.

Koerts, J., Meijer, H. A., Colman, K. S., Tucha, L., Lange, K. W., \& Tucha, O. (2012). What is measured with verbal fluency tests in parkinson's disease patients at different stages of 
402

403

404

405

406

407

408

409

410

411

412

413

414

415

416

417

418

419

420

421

the disease? Journal of Neural Transmission, 120(3), 403-411.

https://doi.org/10.1007/s00702-012-0885-9

Nasreddine, Z. S., Phillips, N. A., Bédirian, V. Ã., Charbonneau, S., Whitehead, V., Collin, I., Cummings, J. L., \& Chertkow, H. (2005). The Montreal Cognitive Assessment, MOCA: A brief screening tool for mild cognitive impairment. Journal of the American Geriatrics Society, 53(4), 695-699. https://doi.org/10.1111/j.1532-5415.2005.53221.x

Ojeda, N., Sánchez, P., Peña, J., Elizagárate, E., Yoller, A. B., Larumbe, J., Gutiérrez, M., Casais, L., \& Ezcurra, J. (2010). Verbal fluency in schizophrenia. Journal of Nervous \& Mental Disease, 198(4), 286-291. https://doi.org/10.1097/nmd.0b013e3181d61748

Open Source Initiative. (n.d.). The MIT License. The MIT License | Open Source Initiative. Retrieved September 23, 2021, from https://opensource.org/licenses/MIT.

Pakhomov, S. V. S., \& Hemmy, L. S. (2014). A computational linguistic measure of clustering behavior on semantic verbal fluency task predicts risk of future dementia in the nun study. Cortex, 55, 97-106. https://doi.org/10.1016/j.cortex.2013.05.009

Shao, Z., Janse, E., Visser, K., \& Meyer, A. S. (2014). What do verbal fluency tasks measure? predictors of verbal fluency performance in older adults. Frontiers in Psychology, 5. https://doi.org/10.3389/fpsyg.2014.00772

Troyer, A. K., Moscovitch, M., \& Winocur, G. (1997). Clustering and switching as two components of verbal fluency: Evidence from younger and older healthy adults. Neuropsychology, 11(1), 138-146. https://doi.org/10.1037/0894-4105.11.1.138 
422 Vance, D., Fazeli, P., Shacka, J., Nicholson, W., McKie, P., Raper, J., Azuero, A., Wadley, V., \&

423 Ball, K. (2017). Testing a computerized cognitive training protocol in adults aging with

424 HIV-associated neurocognitive disorders: Randomized controlled trial rationale and

425 Protocol. JMIR Research Protocols, 6(4). https://doi.org/10.2196/resprot.6625

426 Zemla, J. C., Cao, K., Mueller, K. D., \& Austerweil, J. L. (2020). SNAFU: The Semantic

$427 \quad$ Network and fluency utility. Behavior Research Methods, 52(4), 1681-1699.

428 https://doi.org/10.3758/s13428-019-01343-w 